\title{
Modeling of the signal induced by the charged particles in silicon detector
}

\author{
Przemysław Kulig ${ }^{1, a}$ \\ ${ }^{1}$ Institute of Physics, Jagiellonian University, Krakow, Poland
}

\begin{abstract}
Verification and extensions of the Gaussian cloud dynamics model [1] for the induced current signal in silicon detectors are presented. The approach is based on RamoShockley theorem where, in addition to electrodes field, Coulomb interactions between electron and hole clouds are considered. The preliminary results provide good description of subtle experimental observations gathered by FAZIA collaboration concerning Pulse Shape Analysis (PSA). Focus is put on ion identification and on the factors impacting this mechanism.
\end{abstract}

\section{Introduction and model description}

Particles and fragments identification is of critical importance for nuclear physics. Telescope method is still commonly used in significant number of experiments. That is ruled by energy loss described by Bragg curve. Theory was given in 1930-1933 by Hans Bethe:

$$
-\frac{d E}{d x}=\frac{4 \pi e^{4} z^{2} \rho N_{A} Z}{m_{e} v^{2} A} \ln \left(\frac{2 m_{e} v^{2}}{I}\right)
$$

where $z$ - particle charge, $\rho$ - material density, $N_{A^{-}}$Avogadro number, $Z$ - material atomic number, $m_{e}$ - rest mass electron, $v$ - velocity of particle, $I$ - excitation potential.

But in recent years much attention is paid to Pulse Shape Analysis method which is governed by Plasma Delay Effect. That approach was proposed in 1963 [2] and described formally in 1968 [3] importance of carriers collection times was then highlighted. Charged particles enter silicon detector and create plasma column which length and local charge density are related to Z, mass and particle energy. Pulse rise time (correlated via preamplifier factor to collection time) depends on ionization column dispersion time and drift time of carriers.

That is very important to describe mechanism of processes inside the detector in order to give some recommendations about detector construction. [1] presents the model where Gaussian clouds are used for carriers propagation representation. They are introduced to handle large number of carriers creating complex multibody system and to conduct some analytical calculations. Gaussian centroids are ruled by drift and Gaussian variances are influenced by diffusion and drift. Reverse mode of detector is taken for model considerations as it might give better results in PSA method [4, 5]. That leads to following static field formula:

\footnotetext{
a e-mail: przemyslaw.kulig@uj.edu.pl
} 


$$
E_{\text {static }}(x)=\frac{2 V_{d} x}{d_{S i}^{2}}+\frac{V-V_{d}}{d_{S i}}
$$

where $V$ - bias voltage, $V_{d^{-}}$depletion voltage and $V>V_{d}$.

[1] uses quantum molecular dynamic approach for defining of inter-clouds potential [6]:

$$
\begin{gathered}
v\left(r_{01}, r_{02}, \sigma_{1}, \sigma_{2}\right)=\frac{e^{2} Z_{1} Z_{2}}{\left(2 \pi \sigma_{1} \sigma_{2}\right)^{3}} \iint \frac{\exp \left(\frac{-\left(\mathbf{r}_{1}-\mathbf{r}_{01}\right)^{2}}{2 \sigma_{1}^{2}}\right) \exp \left(\frac{-\left(\mathbf{r}_{2}-\mathbf{r}_{02}\right)^{2}}{2 \sigma_{2}^{2}}\right)}{\left|\mathbf{r}_{1}-\mathbf{r}_{2}\right|} d^{3} \mathbf{r}_{1} d^{3} \mathbf{r}_{2}= \\
=e^{2} Z_{1} Z_{2} \frac{\operatorname{erf}\left(\frac{r_{12}}{\sqrt{2} \sigma}\right)}{r_{12}}
\end{gathered}
$$

where $Z_{1,2^{-}}$charges disribution centered at a relative distance $r_{12}=\left|\mathbf{r}_{01}-\mathbf{r}_{02}\right|$ with variances $\sigma_{1}^{2}$, $\sigma_{2}^{2}$ and $\sigma=\sqrt{\sigma_{1}^{2}+\sigma_{2}^{2}}$.

\section{Optimization and conclusions}

Model verification started with calculation for Boron isotopes. Results are presented in figure 1 and one can notice typical experimental shape with back-bending at lower energies. That point is of high importance as below that level identification ability in PSA method is getting weak. To check if that

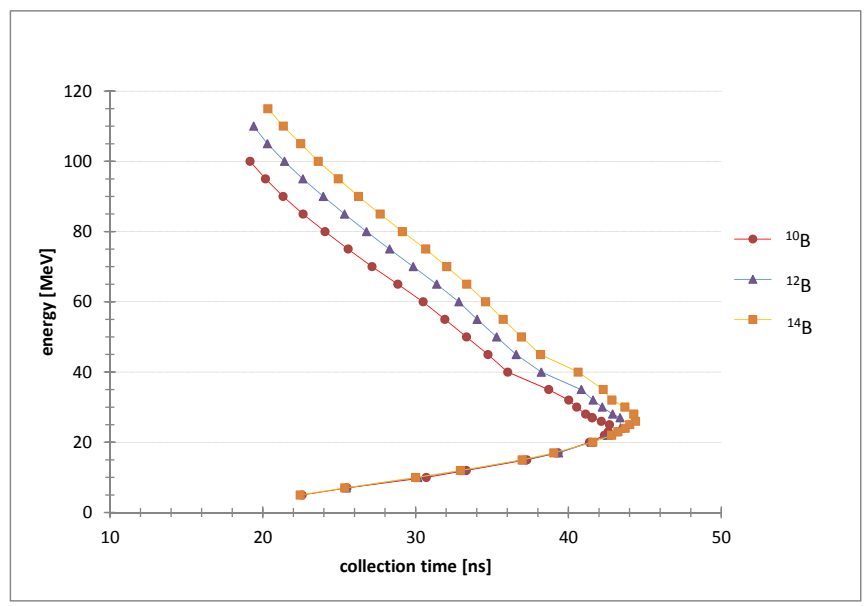

Figure 1. Model calculation for Boron isotope ${ }^{10} B,{ }^{12} B,{ }^{14} B$.

can be influenced in order to improve resolution ability, impact of carrier variance mobility and cariers mobility was verified. The only free parameter of [1] is electron variance mobility $\mu_{\sigma e}$, which is an equivalent of normal mobility in drift process. Originally it was set to $2\left[\frac{\mu m^{2}}{V n s}\right]$.

For verification $\mu_{\sigma e}$ was set also to $0,1,4\left[\frac{\mu m^{2}}{V n s}\right]$ and we used model formula to calculate holes variance mobility:

$$
\mu_{\sigma h}=\mu_{\sigma e} \frac{\mu_{x h}}{\mu_{x e}}
$$

where $\mu_{x e}$ - electrons mobility, $\mu_{x h}$ - holes mobility (material constants). 


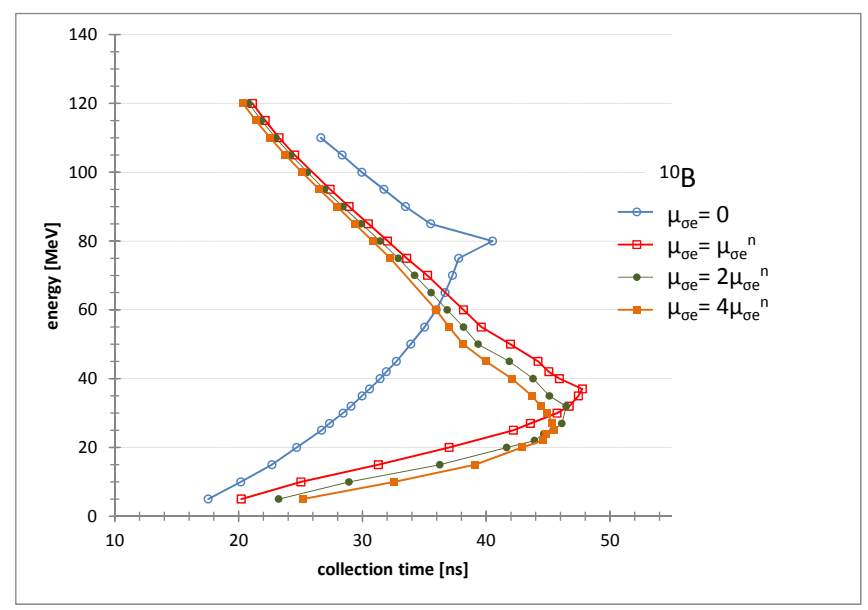

Figure 2. Verification how carriers variance mobility impacts system behaviour $\left(\mu_{\sigma e}^{n}=2\left[\frac{\mu m^{2}}{V n s}\right]\right)$.

Figure 2 shows back-bending point moves to lower energies for increasing $\mu_{\sigma e}$, which means elements identification possible in wider energetical range.

System response to different carriers mobilities was also checked. So far these parameters were set: $\mu_{x e}=135\left[\frac{\mu m^{2}}{V n s}\right] ; \mu_{x h}=47.5\left[\frac{\mu m^{2}}{V n s}\right]$. Figure 3 presents results for doubled and half of original values. One can notice smaller values result in larger collection times and back-bending point appears in smaller energies. That leads to conclusion the research to create material of lowest possible carriers mobility may give a significant improvement in a resolution ability.

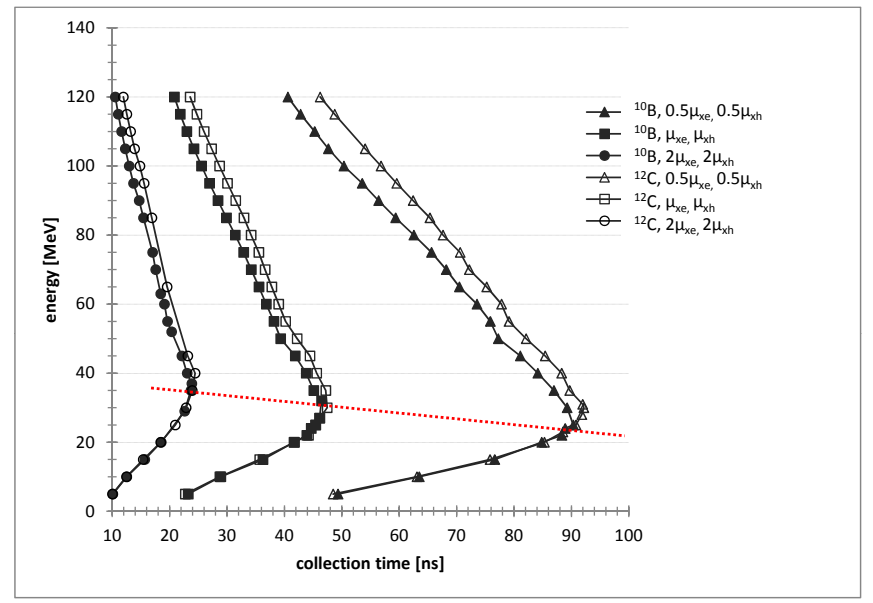

Figure 3. Verification of system behaviour for different values of electrons and holes mobility. Smaller values result in longer collection times and back-bending point appears in smaller energies. 
Similar results for different diffusion coefficients are presented in figure 4 - smaller values of diffusion coefficient makes beck-bending point moving to lower energies which suggests possibility for resolution improvement at lower temperatures.

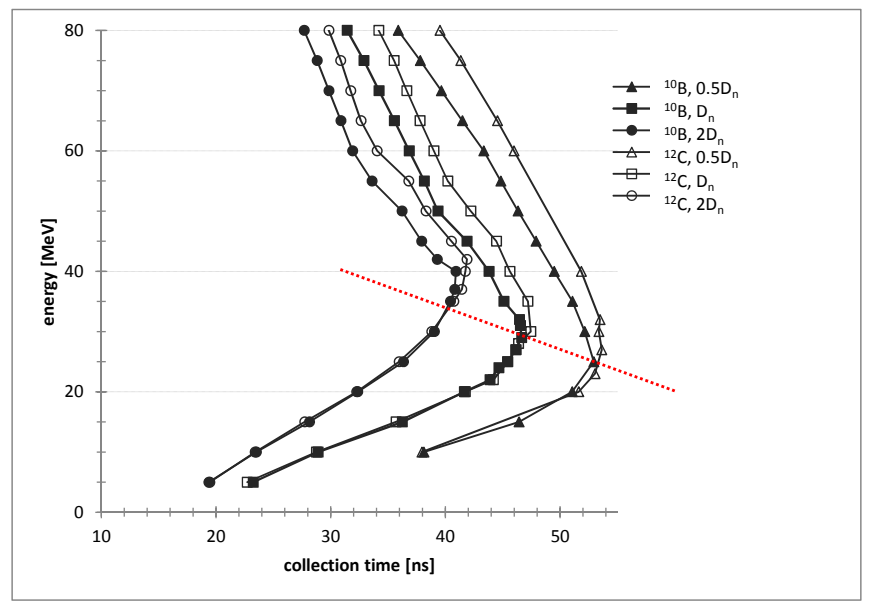

Figure 4. Verification how different diffusion coefficients influence system behaviour. $D_{n}$ is a diffusion cofficient in normal conditions.

That discussion shows identification energy threshold may be expanded when system characteristics (mobilities and diffusion coefficients) are influenced so that carrier collection times is getting longer. In forthcoming papers other parameters and model enhancements will be considered - voltage modification, special doping and temperature dependencies on carrier mobilities as well as wider range of detector thickness.

\section{References}

[1] Z. Sosin, Nuc. Instr. and Meth. in Phys. Res. A 693 170-178 (2012)

[2] C.A. Ammerlaan, et al., Nucl. Instr. and Meth. 22189 (1963)

[3] A. Alherigi Quaranta, A. Taroni, G. Zanarini, IEEE Trans. Nucl. Sci. NS-15, 373 (1968)

[4] H. Hamrita, et al., Nucl. Instr. and Meth. A 531607 (2004)

[5] N. Le Neindre, et al., Nucl. Instr. and Meth. A 701 145-152 (2013)

[6] M. Papa et al., Phys. Rev. C 64024612 (2001) 